\title{
Comparable oncologic outcome of minimally invasive pancreaticoduodenectomy for common bile duct cancer compared with open pancreaticoduodenectomy: A propensity score matched analysis
}

Kyungchul LEE', Woohyung LEE', Yejong PARK', Jae Woo KWON', Eunsung JUN', Ki Byung SONG', Jae Hoon LEE', Seo Young PARK'르. Song Cheol KIM*1

'Division of Hepatobiliary and Pancreatic Surgery, Department of Surgery, University of Ulsan College of Medicine, Asan Medical Center, Seoul, Korea ${ }^{2}$ Department of Clinical Epidemiology and Biostatistics, University of Ulsan College of Medicine, Asan Medical Center, Seoul, Korea

Introduction: Although minimally invasive pancreaticoduodenectomy (MIPD) is expanded from benign to malignant disease, oncologic outcome of MIPD for common bile duct cancer (CBDC) were not confirmed yet.

Methods: The patients who underwent PD for CBDC were identified from 2015 to 2019 in a tertiary referral center. They were divided into open PD (OPD) $(\mathrm{n}=342)$ and MIPD $(\mathrm{n}=87)$ and perioperative and oncologic outcomes were compared between two groups after propensity score matched (PSM) analysis including patients' characteristics, pathology and adjuvant therapy.

Results: OPD group showed higher T stage $(p<0.001)$ and frequent adjuvant chemotherapy $(p=0.017)$ compared with MIPD before PSM and discordance between OPD $(n=78)$ and MIPD $(n=78)$ groups were matched with standardized mean difference $<0.2$ after PSM. There were no differences in terms of overall survival (hazard ratio [HR] $0.538,95 \%$ confidence interval [CI] $0.256-1.132, p=$ 0.102 ) and recurrence free survival (HR $0.798,95 \%$ CI $0.494-1.288, p=0.355)$ after PSM. Hospital stay was comparable $(p=0.631)$ and operative time was longer in MIPD group $(p<0.001)$.

Conclusions: MIPD group showed similar oncologic outcome compared with OPD group after matching of pathologic stage and adjuvant therapy. MIPD could be considerable treatment option for CBDC without oncological compromise in experienced hands. 